\title{
Automated synthesis of control configurations for process networks based on structural coupling
}

\author{
Seongmin $\mathrm{Heo}^{\mathrm{a}}$, W. Alex Marvin ${ }^{\mathrm{b}}$, Prodromos Daoutidis ${ }^{\mathrm{a}, *}$ \\ ${ }^{a}$ Department of Chemical Engineering and Materials Science, University of Minnesota, \\ Minneapolis, MN 55455, USA \\ ${ }^{b}$ BASF Corporation, 500 White Plains Road, Tarrytown, NY 10591, USA
}

\begin{abstract}
In this paper, a method to systematically synthesize control configurations with favorable structural coupling is developed, using relative degree as a measure of such coupling. Initially, an integer optimization problem is formulated to identify optimal distributions of inputs and outputs (decentralized control configurations) that minimize the overall structural coupling in the network. Then, a hierarchical clustering procedure, which allows identifying groups of inputs and outputs that are strongly connected topologically (block decentralized control configurations), is proposed. The application of the method is illustrated through an example process network.
\end{abstract}

Keywords: Control structure selection, Plantwide control, Process networks, Integer programming, Hierarchical clustering

\section{Introduction}

Control structure design, i.e. the selection and pairing of manipulated inputs and controlled outputs, is a classic problem in control that has received a lot of attention in the literature (see e.g. van de Wal and de Jager, 2001). In process control in particular, this problem has been studied extensively in the context of plantwide control design (see e.g. Rangaiah and Kariwala, 2012). Examples include: (a) heuristics-based approaches whereby the selection and pairing are perfomed following logical rules (e.g. Price and Georgakis, 1993; Ng and Stephanopoulos, 1996; Luyben et al., 1997); (b) the concept of self-optimizing control for selecting controlled outputs

\footnotetext{
*Corresponding author.

Email address: daout001@umn.edu (Prodromos Daoutidis)
}

Preprint submitted to Elsevier

January 28, 2015

(C) 2015. This manuscript version is made available under the Elsevier user license http://www.elsevier.com/open-access/userlicense/1.0/ 
as the basis for control structure design (e.g. Skogestad, 2000a,b; Baldea et al., 2008); (c) the concept of relative gain array (RGA) (e.g. Chang and Yu, 1990) and block RGA (e.g. Manousiouthakis et al., 1986; Kariwala et al., 2003); and (d) optimization based approaches using for example economic criteria (e.g. Heath et al., 2000; Psaltis et al., 2013; Ellis and Christofides, 2014; Psaltis et al., 2014).

The problem of control structure selection becomes particularly challenging for tighly integrated process networks, which are the rule rather than the exception in modern chemical and energy plants, and also in the context of smart manufacturing (Christofides et al., 2007). Integration results in significant economic benefits, but also limits the available degrees of freedom and leads to significant interactions that need to be addressed in controller design (including the selection of the control configuration). In a series of papers (Kumar and Daoutidis, 2002; Baldea et al., 2006; Jogwar et al., 2009), we have documented that whenever integration results in large rates of recovery and recycle of material and/or energy (compared to the input/output flows), a time-scale hierarchy develops: individual units evolve in a fast time scale (and are affected by the large internal and recycle flows) and slower network-level dynamics emerge (that are affected by the small external flows). This particular feature can be exploited for the selection of manipulated inputs acting in appropriate time scales to address individual process control objectives and network level objectives, in the context of hierarchical control.

However, integrated process networks are not necessarily characterized by a segregation of material and/or energy flows. The design of control systems for such networks is a challenging, open problem, that has been addressed, for example, using passivity-based control (e.g. Ydstie, 2002; Hudon and Bao, 2012; Tippett and Bao, 2013), distributed control (e.g. Rawlings and Stewart, 2008; Liu et al., 2009; Scattolini, 2009) and quasi-decentralized control (e.g. Mhaskar et al., 2007; Sun and El-Farra, 2008). Central to this problem is the pairing of manipulated inputs and controlled outputs, either in the form of single-input single-output controllers or multivariable ones. A promising approach to this end is to exploit the extensive work in network theory and graph theory towards identifying "communities" (of manipulated inputs and controlled outputs, in our case) whose members interact strongly among them, yet are weakly coupled to the rest of the network members (e.g. Girvan and Newman, 2002; Jiang et al., 2007). This community detection problem can be pursued using optimization of clustering parameters that capture the network connectivity (e.g. Newman, 2004, 2006) or through spectral graph theory (e.g. Varigonda et al., 2004). The former approaches are generally more easily scalable to larger networks.

For the pairing of manipulated inputs and controlled outputs, which we will 
refer to as the control configuration synthesis problem, a meanigful approach is to seek potential inputs and outputs that are strongly connected topologically. One measure of topological closeness is the concept of relative degree (Daoutidis and Kravaris, 1992). Relative degree essentially captures the directness of the effect of an input on an output, or the physical closeness between the two variables, and can be used to identify input/output clusters with favorable "structural coupling" in the above sense (Daoutidis and Kravaris, 1992; Schné and Hangos, 2011). Its generic calculation requires only structural information on the dynamic interactions in the network, and can be automated on the basis of an equation graph that captures these dynamic interactions.

In this paper, we develop a method to systematically synthesize control configurations with favorable structural coupling, using relative degree as a measure of such coupling. Initially, we formulate an integer optimization problem to identify optimal distributions of inputs and outputs (essentially decentralized control configurations) that minimize the overall structural coupling in the network. We then propose a hierarchical clustering procedure which allows identifying groups of inputs and outputs that are strongly connected topologically, and are thus block decentralized control configuration candidates. The proposed approach is flexible as it allows generating control configurations that span the entire gamut from fully decentralized ones to the fully centralized one. It can also be automated for ease of implementation. Its application is illustrated through a case study on an integrated energy system.

\section{Relative degree as a measure of structural coupling}

Let us consider a general state space model of the form:

$$
\begin{gathered}
\dot{x}=f(x)+\sum_{i=1}^{n_{u}} g_{i}(x) u_{i} \\
y_{j}=h_{j}(x), j=1, \ldots, n_{y}
\end{gathered}
$$

where $x \in \mathbb{R}^{n_{x}}$ denotes the state variables, and $u_{i}, y_{j} \in \mathbb{R}$ denote the input variables and the output variables, respectively. $f, g_{i}$ are vector fields on $\mathbb{R}^{n_{x}}$, and $h_{j}$ are scalar fields on $\mathbb{R}^{n_{x}}$.

The relative degree between $u_{i}$ and $y_{j}, r_{i j}$, is defined as the smallest integer that satisfies (Khalil, 2002):

$$
\mathcal{L}_{g_{i}} \mathcal{L}_{f}^{r_{i j}-1} h_{j}(x) \not \equiv 0
$$


where $\mathcal{L}$ represents the Lie derivative, defined as:

$$
\mathcal{L}_{f(x)} h(x)=\frac{\partial h(x)}{\partial x} f(x)
$$

In Daoutidis and Kravaris (1992), it was shown that the relative degree can be intepreted as a measure of how direct the effect of an input is on an output, as it represents the number of integrations needed for an input to affect an output. It was also argued that the relative degree can be used as a measure of physical closeness between an input and an output as it captures the sluggishness of the input/output response (in effect representing an apparent dead time). Also, it was shown that the generic calculation of relative degree requires only structural information of a process, i.e. knowledge of the interdependencies among the process variables. This can be used to construct an equation graph where nodes represent the state, input and output variables, and edges represent the interdependencies among the process variables. Edges are added based on the following rules:

- there is an edge from node $x_{k}$ to node $x_{l}$ if $\partial f_{l}(x) / \partial x_{k} \not \equiv 0$

- there is an edge from node $u_{i}$ to node $x_{l}$ if $g_{i l}(x) \not \equiv 0$

- there is an edge from node $x_{k}$ to node $y_{j}$ if $\partial h_{j}(x) / \partial x_{k} \not \equiv 0$

where $f_{l}(x), g_{i l}(x)$ are the $l$-th element of $f(x), g_{i}(x)$, respectively.

In such a graph, a path is defined as an open walk of nodes and edges of a graph such that no node is repeated. The length of a path is the number of edges contained in the path. An input-to-output path (IOP) is a path which starts from an input node and terminates at an output node. The relative degree $r_{i j}$ is then related to the length of the shortest IOP connecting $u_{i}$ and $y_{j}$ of the equation graph as follows (Daoutidis and Kravaris, 1992):

$$
r_{i j}=l_{i j}-1
$$

Based on the above, relative degree can be used as a measure of structural coupling, i.e. coupling among the input/output variables based on their structural interdependencies, to provide guidelines for the design and evaluation of multi-loop control configurations. The procedure described in Daoutidis and Kravaris (1992) involves the following steps:

1. Compute the relative degrees between all the inputs and the outputs to form a relative degree matrix $(\mathrm{RDM})$ whose elements are the relative degrees between 
the inputs and the outputs:

$$
M_{r}=\left[\begin{array}{ccc}
r_{11} & \cdots & r_{1 n_{y}} \\
\vdots & \ddots & \vdots \\
r_{n_{u} 1} & \cdots & r_{n_{u} n_{y}}
\end{array}\right]
$$

2. Rearrange the outputs such that the minimum relative degree in each column of the RDM falls on the major diagonal. Then, the diagonal elements of the rearranged RDM represent the relative degrees between the input/output pairs forming the control configuration with a favorable structural coupling. The off-diagonal relative degrees in a row capture the coupling between a specific input and the other outputs, while the off-diagonal relative degrees in a column capture the coupling between a specific output and the other inputs.

3. Evaluate the overall structural coupling for the particular input/output assignment by comparing the diagonal and the off-diagonal relative degrees. Specifically, the differences between off-diagonal and diagonal relative degrees in a row (i.e. $r_{i j}-r_{i i}$ ) and in a column (i.e. $r_{j i}-r_{i i}$ ) provide a measure of the overall structural coupling. The larger these differences are, the more favorable the control configuration is.

The above procedure is time consuming to apply to large scale process networks as each step needs to be executed manually. Also, only fully decentralized control configurations are considered. In what follows, we exploit the graph theoretic interpretation of relative degrees to propose a generally applicable and easy to automate framework to synthesize optimal control configurations (ranging from fully decentralized to fully centralized) for process networks.

\section{Control configuration synthesis based on structural coupling}

In this section, we develop an optimization-based framework to synthesize control configurations based on structural coupling. We define a control configuration synthesis problem as a distribution problem, where the inputs and the outputs are distributed to the controllers. The assumption is that the controllers are square, i.e. the same numbers of inputs and outputs are distributed to each controller. We define the size of a controller as the number of inputs (or outputs) that are distributed to the controller. Initially, we limit the size of all the controllers to 1 (i.e. we consider fully decentralized control configurations only), and formulate an optimization problem to generate such decentralized control configurations so as to minimize the 
overall structural coupling between the different loops. Starting from such decentralized control configurations, we then describe the synthesis of block decentralized control configurations by hierarchical clustering of the input/output pairs.

\subsection{Synthesis of decentralized control configurations}

Let us consider a system with $n_{u}$ inputs and $n_{y}$ outputs, and the corresponding RDM, $M_{r}$. We assume that we have more inputs than outputs which is typically the case in practical problems (i.e. $n_{u}>n_{y}$ ). For a given input/output assignment, consider the RDM rearranged such that the relative degrees between the chosen input/output pairs fall on the main diagonal. The structural coupling of this configuration can be evaluated using the differences between the diagonal and off-diagonal relative degrees of the first $n_{y}$ rows of the RDM, and noting that the relative degrees in the remaining $n_{u}-n_{y}$ rows do not affect the structural coupling of the system as they represent the relative degrees between the unselected inputs (i.e. the inputs that are not distributed to any controller) and the outputs. The overall structural coupling for the given control configuration can thus be evaluated based on the value of the following term:

$$
J_{D C}=\sum_{i=1}^{n_{y}} \sum_{j=1}^{n_{y}}\left(r_{i j}-r_{i i}\right)
$$

The larger the above term is (i.e. the smaller the diagonal relative degrees and the larger the off-diagonal ones), the more favorable the control configuration is.

We note that the relative degrees in a column can be classifed into three classes, diagonal $\left(\mathbf{r}_{d}\right)$, off-diagonal $\left(\mathbf{r}_{o}\right)$ and the remaining ones $\left(\mathbf{r}_{r}\right)$. The relative degree $r_{i j}$ is:

- in $\mathbf{r}_{d}$ if $u_{i}$ and $y_{j}$ are distributed to the same controller

- in $\mathbf{r}_{r}$ if $u_{i}$ is not distributed to any controller

- in $\mathbf{r}_{o}$ otherwise

Let us now define two different sets of binary variables that will allow us to capture this classification without rearranging the RDM:

$$
\begin{aligned}
p_{i j} & = \begin{cases}1, & \text { if } u_{i} \text { is distributed to the same controller with } y_{j} \\
0, & \text { otherwise }\end{cases} \\
q_{i} & = \begin{cases}0, & \text { if } u_{i} \text { is not distributed to any controller } \\
1, & \text { otherwise }\end{cases}
\end{aligned}
$$


Note that, by definition, $p_{i j}$ cannot be equal to 1 if $q_{i}$ is equal to 0 , leaving three possible combinations of $p_{i j}$ and $q_{i}$, corresponding to the classes of relative degrees defined above:

$$
\begin{aligned}
& \mathbf{r}_{d}=\left\{r_{i j} \in M_{r} \mid p_{i j}=1, q_{i}=1\right\} \\
& \mathbf{r}_{o}=\left\{r_{i j} \in M_{r} \mid p_{i j}=0, q_{i}=1\right\} \\
& \mathbf{r}_{r}=\left\{r_{i j} \in M_{r} \mid p_{i j}=0, q_{i}=0\right\}
\end{aligned}
$$

Also note that, for systems with more outputs than inputs, $q_{j}$, instead of $q_{i}$, can be defined such that $q_{j}$ is equal to 0 if $y_{j}$ is not distributed to any controller.

The optimization problem for decentralized control configuration synthesis corresponds to an exhaustive search over all possible input/output distributions such that the term in Eq.(4) is maximized. Using the binary variables defined in Eqs.(5)-(6), this optimization problem can be stated as follows:

$$
\operatorname{maximize} J_{D C}=\sum_{i=1}^{n_{u}} \sum_{j=1}^{n_{y}} r_{i j} q_{i}-n_{y} \sum_{i=1}^{n_{u}} \sum_{j=1}^{n_{y}} r_{i j} p_{i j}
$$

where the first and the second terms of the objective function represent the corresponding terms in Eq.(4), and the decision variables are $p_{i j}$ and $q_{i}$. We now describe a set of constraints to complete the problem.

The number of input/output pairs needs to be equal to the number of outputs to ensure that all the outputs are distributed to a controller:

$$
\sum_{i, j} p_{i j}=n_{y}
$$

The following set of constraints states that any input or output can be distributed to one controller at most:

$$
\begin{aligned}
& \sum_{j} p_{i j} \leq 1 \forall i \\
& \sum_{i} p_{i j} \leq 1 \forall j
\end{aligned}
$$

Finally, since any input $u_{i}$ can be distributed to at most one output $y_{i}$ :

$$
q_{i} \leq \sum_{j} p_{i j} \forall i
$$


This formulation (Eqs.(7)-(11)) corresponds to a bipartite matching problem Leiserson et al. (2001), where a set of variables is divided into two disjoint subsets (in this case, the set of inputs and the set of outputs), and the variables from each set are matched so as to minimize/maximize the objective function. For such a problem, the existence of a global optimum is guaranteed, although in general there does not exist a unique solution (Leiserson et al., 2001). This optimization problem can be solved within the environment of GAMS using the CPLEX Optimizer (IBM, 2011). We can also find alternative solutions with the same objective value by excluding the optimal solutions that have been already found and resolving the optimization problem.

The output of the optimization problem is the $n_{u} \times n_{y}$ matrix $P_{\text {opt }}$ whose $i, j$-th element is $p_{i j}$. Note that $P_{o p t}$ is a variant of a permutation matrix whose $i$-th row sum is 1 if $u_{i}$ is distributed to a controller, and 0 otherwise. Using $P_{o p t}$, we can obtain the optimal RDM $\left(n_{y} \times n_{y}\right), M_{r, o p t}$, as:

$$
M_{r, o p t}=P_{o p t}^{T} M_{r}
$$

Note that, by premultiplying $M_{r}$ by $P_{o p t}^{T}$, we eliminate the $n_{u}-n_{y}$ rows of $M_{r}$, whose elements represent the relative degrees between the unselected inputs and the outputs, while rearranging the remaining $n_{y}$ rows with respect to the optimal decentralized control configuration.

Example. For a simple illustration, let us consider a continuous stirred tank reactor (CSTR) shown in Figure 1. Feed streams of $\mathrm{A}$ and $\mathrm{B}$ with molar flow rates $F_{A}$, $F_{B}$, temperatures $T_{A}, T_{B}$, and concentrations $c_{A 0}, c_{B 0}$, respectively, are fed to the reactor, where a reaction $A+B \rightarrow C+D$ occurs, to produce a product stream with molar flow rate $F$, temperature $T$, and concentrations $c_{A}, c_{B}, c_{C}, c_{D}$. We assume that the reaction is endothermic, and heat is supplied to the reactor at a rate $Q$ to facilitate the reaction.

Assuming constant density $\rho$ and heat capacity $C_{p}$, the energy/material balances 
of the system can be described by the following equations:

$$
\begin{aligned}
\frac{d M}{d t} & =F_{A}+F_{B}-F \\
\frac{d c_{A}}{d t} & =\frac{F_{A}}{M}\left(c_{A 0}-c_{A}\right)-\frac{F_{B}}{M} c_{A}-k c_{A} c_{B} \exp \left(\frac{-E}{R T}\right) \\
\frac{d c_{B}}{d t} & =\frac{F_{B}}{M}\left(c_{B 0}-c_{B}\right)-\frac{F_{A}}{M} c_{B}-k c_{A} c_{B} \exp \left(\frac{-E}{R T}\right) \\
\frac{d c_{C}}{d t} & =-\frac{F_{A}+F_{B}}{M} c_{C}+k c_{A} c_{B} \exp \left(\frac{-E}{R T}\right) \\
\frac{d T}{d t} & =\frac{F_{A}}{M}\left(T_{A}-T\right)+\frac{F_{B}}{M}\left(T_{B}-T\right)+\frac{Q}{M C_{p}}-\frac{\Delta H}{\rho C_{p}} k c_{A} c_{B} \exp \left(\frac{-E}{R T}\right)
\end{aligned}
$$

where $M$ is the molar holdup of the reactor, and $k$ and $E$ are the rate constant and the activation energy of the reaction. $\Delta H$ represents the heat of reaction.

We define the following sets of the inputs and the outputs:

$$
\begin{aligned}
& \mathbf{u}=\left\{F_{A}, F_{B}, F, Q\right\} \\
& \mathbf{y}=\left\{M, c_{A}, c_{C}, T\right\}
\end{aligned}
$$

Then, the equation graph of the system can be obtained as shown in Figure 2. The RDM of the system can be computed as follows:

$$
M_{r}=\begin{aligned}
& M \\
& F_{A} \\
& F_{B} \\
& F \\
& Q
\end{aligned}\left[\begin{array}{cccc}
1 & 1 & 1 & 1 \\
1 & 1 & 1 & 1 \\
1 & 2 & 2 & 2 \\
\infty & 2 & 2 & 1
\end{array}\right]
$$

and the shortest IOPs between all input/output pairs are provided in Table 1.

By solving the optimization problem defined by Eqs.(7)-(11), we get two optimal solutions as follows:

$$
\begin{aligned}
P_{o p t, 1} & =\left[\begin{array}{llll}
0 & 1 & 0 & 0 \\
0 & 0 & 1 & 0 \\
1 & 0 & 0 & 0 \\
0 & 0 & 0 & 1
\end{array}\right] \\
P_{o p t, 2}= & {\left[\begin{array}{llll}
0 & 0 & 1 & 0 \\
0 & 1 & 0 & 0 \\
1 & 0 & 0 & 0 \\
0 & 0 & 0 & 1
\end{array}\right] }
\end{aligned}
$$


which translate into the following optimal RDMs:

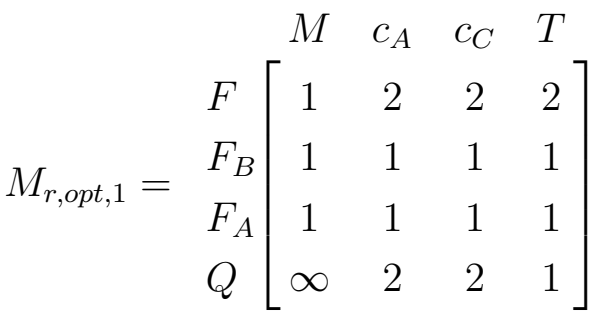

$$
\begin{aligned}
& \begin{array}{lllll}
M & c_{A} & c_{C} & T
\end{array} \\
& M_{r, \text { opt }, 2}=\begin{array}{l}
F \\
F_{A} \\
F_{B} \\
Q
\end{array}\left[\begin{array}{cccc}
1 & 2 & 2 & 2 \\
1 & 1 & 1 & 1 \\
1 & 1 & 1 & 1 \\
\infty & 2 & 2 & 1
\end{array}\right]
\end{aligned}
$$

In both control configurations, the molar holdup and the temperature of the reactor is controlled by manipulating the outlet flowrate and the heat input rate, respectively. The flowrates of the feed streams of $A$ and $B$ are respectively manipulated to control the concentrations of $C$ and $A$ of the outlet stream in the control configuration given by $M_{r, o p t, 1}$, and to control the concentrations of $A$ and $C$ of the outlet stream in the control configuration given by $M_{r, o p t, 2}$.

\subsection{Hierarchical clustering of input/output pairs}

In this subsection, we propose a systematic way to cluster the input/output pairs hierarchically, by considering the structural coupling between different input/output pairs (rather than simply inputs and outputs). The goal is to generate a hierarchy of block decentralized control configurations with increasing controller sizes, whereby in each level we form clusters of input/output pairs which are structurally close in a sense that will be made precise shortly. The result of clustering can be visualized as a dendrogram where each level of the dendrogram represents a block decentralized control configuration.

Let us consider the following optimal RDM obtained as a solution to the optimization problem discussed above (in the case of multiple optimal solutions, each 
one can be considered separately):

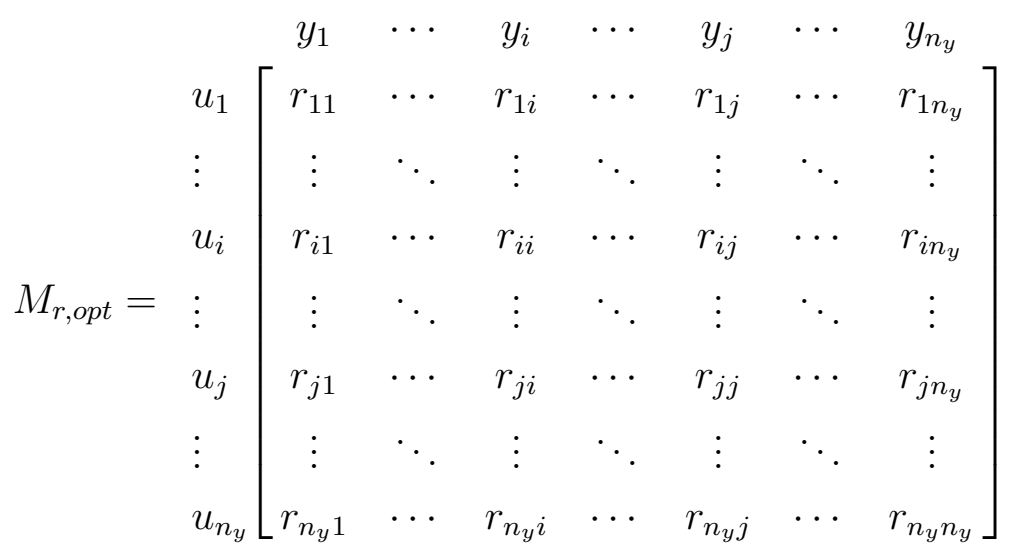

Two input/output pairs, $\left\{u_{i}, y_{i}\right\}$ and $\left\{u_{j}, y_{j}\right\}$, will be structurally close if the inputs $u_{i}, u_{j}$ have comparable structural effect on the outputs $y_{i}, y_{j}$, i.e. the values of the off-diagonal relative degrees $r_{i j}$ and $r_{j i}$ are close to the values of the diagonal relative degrees $r_{i i}$ and $r_{j j}$. Motivated by this, we define a distance between the two input/output pairs as the sum of the largest differences between the off-diagonal and diagonal relative degrees:

$$
\begin{aligned}
d\left(\left\{u_{i}, y_{i}\right\},\left\{u_{j}, y_{j}\right\}\right) & =\left(\max \left\{r_{i j}, r_{j i}\right\}-r_{i i}\right)+\left(\max \left\{r_{i j}, r_{j i}\right\}-r_{j j}\right) \\
& =2 \max \left\{r_{i j}, r_{j i}\right\}-r_{i i}-r_{j j}
\end{aligned}
$$

Proposition 3.1. The distance defined in Eq.(14) is a pseudo-semi-metric (Kunen and Vaughan, 1984), satisfying the following properties:

$$
\begin{aligned}
& d\left(\left\{u_{i}, y_{i}\right\},\left\{u_{j}, y_{j}\right\}\right) \geq 0 \\
& d\left(\left\{u_{i}, y_{i}\right\},\left\{u_{i}, y_{i}\right\}\right)=0 \\
& d\left(\left\{u_{i}, y_{i}\right\},\left\{u_{j}, y_{j}\right\}\right)=d\left(\left\{u_{j}, y_{j}\right\},\left\{u_{i}, y_{i}\right\}\right)
\end{aligned}
$$

Proof. The objective function value computed from the above optimal RDM is:

$$
\begin{aligned}
J_{D C, \text { opt }}= & \sum_{k=1}^{n_{y}} \sum_{l=1}^{n_{y}}\left(r_{k l}-r_{l l}\right) \\
= & {\left[\left(r_{11}-r_{11}\right)+\cdots+\left(r_{1 i}-r_{11}\right)+\cdots+\left(r_{1 j}-r_{11}\right)+\cdots+\left(r_{1 n_{y}}-r_{11}\right)\right]+\cdots } \\
& +\left[\left(r_{i 1}-r_{i i}\right)+\cdots+\left(r_{i i}-r_{i i}\right)+\cdots+\left(r_{i j}-r_{i i}\right)+\cdots+\left(r_{i n_{y}}-r_{i i}\right)\right]+\cdots \\
& +\left[\left(r_{j 1}-r_{j j}\right)+\cdots+\left(r_{j i}-r_{j j}\right)+\cdots+\left(r_{j j}-r_{j j}\right)+\cdots+\left(r_{j n_{y}}-r_{j j}\right)\right]+\cdots \\
& +\left[\left(r_{n_{y} 1}-r_{n_{y} n_{y}}\right)+\cdots+\left(r_{n_{y} i}-r_{n_{y} n_{y}}\right)+\cdots+\left(r_{n_{y} j}-r_{n_{y} n_{y}}\right)+\cdots+\left(r_{n_{y} n_{y}}-r_{n_{y} n_{y}}\right)\right]
\end{aligned}
$$


Let us swap the $i, j$-th rows to get the following RDM:

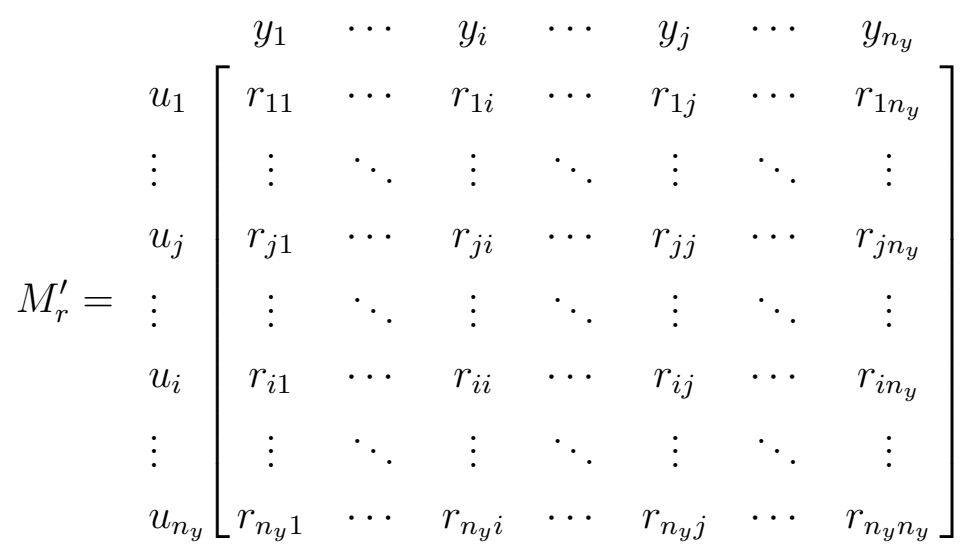

The objective function value computed from the above RDM is:

$$
\begin{aligned}
J_{D C}^{\prime}= & {\left[\left(r_{11}-r_{11}\right)+\cdots+\left(r_{1 i}-r_{11}\right)+\cdots+\left(r_{1 j}-r_{11}\right)+\cdots+\left(r_{1 n_{y}}-r_{11}\right)\right]+\cdots } \\
& +\left[\left(r_{j 1}-r_{j i}\right)+\cdots+\left(r_{j i}-r_{j i}\right)+\cdots+\left(r_{j j}-r_{j i}\right)+\cdots+\left(r_{j n_{y}}-r_{j i}\right)\right]+\cdots \\
& +\left[\left(r_{i 1}-r_{i j}\right)+\cdots+\left(r_{i i}-r_{i j}\right)+\cdots+\left(r_{i j}-r_{i j}\right)+\cdots+\left(r_{i n_{y}}-r_{i j}\right)\right]+\cdots \\
& +\left[\left(r_{n_{y} 1}-r_{n_{y} n_{y}}\right)+\cdots+\left(r_{n_{y} i}-r_{n_{y} n_{y}}\right)+\cdots+\left(r_{n_{y} j}-r_{n_{y} n_{y}}\right)+\cdots+\left(r_{n_{y} n_{y}}-r_{n_{y} n_{y}}\right)\right]
\end{aligned}
$$

The difference between the objective function values is:

$$
\begin{aligned}
J_{D C, \text { opt }}-J_{D C}^{\prime}= & {\left[\left(r_{i 1}-r_{i i}\right)+\cdots+\left(r_{i i}-r_{i i}\right)+\cdots+\left(r_{i j}-r_{i i}\right)+\cdots+\left(r_{i n_{y}}-r_{i i}\right)\right.} \\
& \left.+\left(r_{j 1}-r_{j j}\right)+\cdots+\left(r_{j i}-r_{j j}\right)+\cdots+\left(r_{j j}-r_{j j}\right)+\cdots+\left(r_{j n_{y}}-r_{j j}\right)\right] \\
& -\left[\left(r_{j 1}-r_{j i}\right)+\cdots+\left(r_{j i}-r_{j i}\right)+\cdots+\left(r_{j j}-r_{j i}\right)+\cdots+\left(r_{j n_{y}}-r_{j i}\right)\right. \\
& \left.+\left(r_{i 1}-r_{i j}\right)+\cdots+\left(r_{i i}-r_{i j}\right)+\cdots+\left(r_{i j}-r_{i j}\right)+\cdots+\left(r_{i n_{y}}-r_{i j}\right)\right] \\
= & n\left(r_{i j}+r_{j i}-r_{i i}-r_{j j}\right)
\end{aligned}
$$

Recall that $M_{r, o p t}$ is the optimal RDM, implying that the above difference should be greater than or equal to 0 , i.e.:

$$
n\left(r_{i j}+r_{j i}-r_{i i}-r_{j j}\right) \geq 0
$$

Then,

$$
d\left(\left\{u_{i}, y_{i}\right\},\left\{u_{j}, y_{j}\right\}\right)=2 \max \left\{r_{i j}, r_{j i}\right\}-r_{i i}-r_{j j} \geq r_{i j}+r_{j i}-r_{i i}-r_{j j} \geq 0
$$

The second and third properties can be shown easily from Eq.(14). 
The main idea behind the proposed clustering procedure is to identify the input/output pairs that are structurally close (i.e. have a small distance) and merge them to form a cluster of input/output pairs that will form a new controller block in a block decentralized configuration. At each level of clustering, the number of controllers is decreased by 1 (although more general formulations are possible), and the clustering procedure stops when there is only one controller (i.e. when we have fully centralized control configuration).

Let us first consider the merging of individual input/output pairs. Note that there is always the possibility that there can be multiple pairs with identical distance. Let us consider the following example RDM:

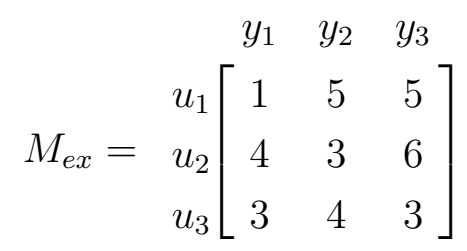

Note that $d\left(\left\{u_{1}, y_{1}\right\},\left\{u_{2}, y_{2}\right\}\right)=d\left(\left\{u_{2}, y_{2}\right\},\left\{u_{3}, y_{3}\right\}\right)=d\left(\left\{u_{1}, y_{1}\right\},\left\{u_{3}, y_{3}\right\}\right)=6$, i.e. all three input/output pairs are equally spaced in terms of the distance defined in Eq.(14). However, different pairs of input/output pairs (e.g. $\left\{u_{1}, y_{1}\right\} /\left\{u_{2}, y_{2}\right\}$ and $\left.\left\{u_{1}, y_{1}\right\} /\left\{u_{3}, y_{3}\right\}\right)$ have different structural coupling. In such a case, we need to impose additional criteria to determine the input/output pairs to be merged. A natural first such criterion is to use the sum of the smallest differences between off-diagonal and diagonal relative degrees, i.e.:

$$
\delta\left(\left\{u_{i}, y_{i}\right\},\left\{u_{j}, y_{j}\right\}\right)=2 \min \left\{r_{i j}, r_{j i}\right\}-r_{i i}-r_{j j}
$$

Additionally, we could use the actual values of the off-diagonal elements to further discriminate between different pairs of input/output pairs. To this end, we define:

$$
\Delta\left(\left\{u_{i}, y_{i}\right\},\left\{u_{j}, y_{j}\right\}\right)=\max \left\{r_{i j}, r_{j i}\right\}
$$

We can now define the distance matrices, $D_{d}, D_{\delta}$ and $D_{\Delta}$, whose $i, j$-th elements are $d\left(\left\{u_{i}, y_{i}\right\},\left\{u_{j}, y_{j}\right\}\right), \delta\left(\left\{u_{i}, y_{i}\right\},\left\{u_{j}, y_{j}\right\}\right)$ and $\Delta\left(\left\{u_{i}, y_{i}\right\},\left\{u_{j}, y_{j}\right\}\right)$, respectively. We can also define a distance triplet as $(d, \delta, \Delta)$. For the example in Eq.(15), $D_{d}, D_{\delta}$ 
and $D_{\Delta}$ take the form:

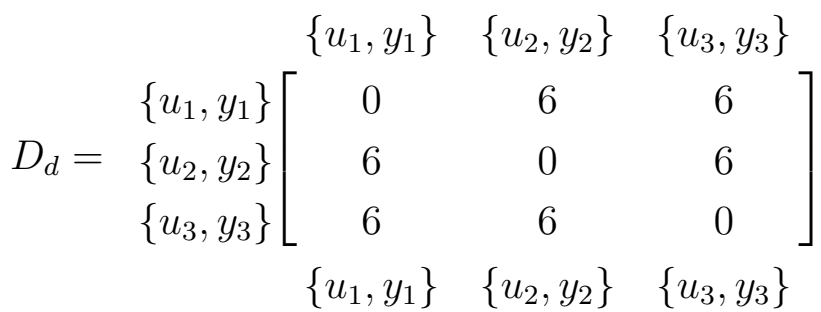

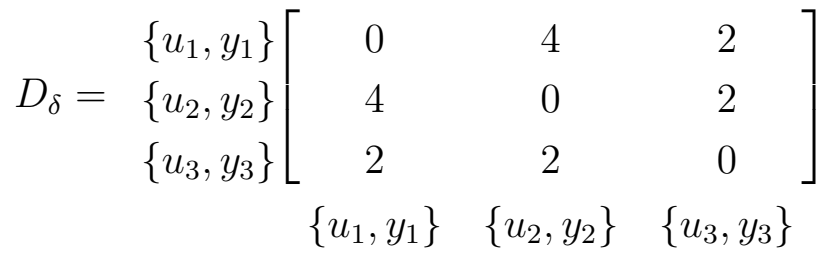

$$
\begin{aligned}
& D_{\Delta}=\begin{array}{l}
\left\{u_{1}, y_{1}\right\} \\
\left\{u_{2}, y_{2}\right\} \\
\left\{u_{3}, y_{3}\right\}
\end{array}\left[\begin{array}{lll}
0 & 5 & 5 \\
5 & 0 & 6 \\
5 & 6 & 0
\end{array}\right]
\end{aligned}
$$

Note that $\delta\left(\left\{u_{1}, y_{1}\right\},\left\{u_{2}, y_{2}\right\}\right)>\delta\left(\left\{u_{2}, y_{2}\right\},\left\{u_{3}, y_{3}\right\}\right)=\delta\left(\left\{u_{1}, y_{1}\right\},\left\{u_{3}, y_{3}\right\}\right)$. Furthermore, $\Delta\left(\left\{u_{2}, y_{2}\right\},\left\{u_{3}, y_{3}\right\}\right)>\Delta\left(\left\{u_{1}, y_{1}\right\},\left\{u_{3}, y_{3}\right\}\right)$. Based on these criteria, $\left\{u_{1}, y_{1}\right\}$ and $\left\{u_{3}, y_{3}\right\}$, with the distance triplet of $(6,2,5)$, are the input/output pairs to be merged into a cluster.

We can now proceed to discuss the merging of input/output clusters. Let us consider the set of all optimal input/output pairs, $I O_{i}=\left\{u_{i}, y_{i}\right\}$, and denote it as $I O$. Any subset of this set will be an input/output cluster, and be denoted by $C(A)$ where $\mathrm{A}$ is the set of indices of the input/output pairs in the cluster (e.g. $\left.C(\{k, l, m\})=\left\{I O_{k}, I O_{l}, I O_{m}\right\}\right)$. Note that a single input/output pair can be considered as a (trivial) cluster by itself (e.g. $\left.C(\{k\})=\left\{I O_{k}\right\}\right)$. The closeness between input/output clusters can be assessed through an exhaustive search over all possible pairs of input/output pairs from the two clusters, such that their distance is maximized. This leads naturally to the following definition of the distance between clusters $C(A)$ and $C\left(A^{\prime}\right)$ :

$$
d\left(C(A), C\left(A^{\prime}\right)\right)=\max \left\{d\left(I O_{k}, I O_{l}\right) \mid k \in A, l \in A^{\prime}\right\}
$$

Note that, in this case too, there is the possibility that there can be multiple pairs of input/output clusters with the same distance. These can be further discriminated using the criteria that we defined previously. Specifically, we can define:

$$
\begin{aligned}
\delta\left(C(A), C\left(A^{\prime}\right)\right) & =\delta\left(I O_{k}, I O_{l}\right) \\
\Delta\left(C(A), C\left(A^{\prime}\right)\right) & =\Delta\left(I O_{k}, I O_{l}\right)
\end{aligned}
$$


where $I O_{k}, I O_{l}$ are the input/output pairs identified in Eq.(18). The corresponding distance matrices, $D_{d}, D_{\delta}$ and $D_{\Delta}$, can then be formed at each step of the procedure and used to determine the clusters to be merged.

For the example in Eq.(15), the distances $d, \delta$ and $\Delta$ between the newly formed cluster, $C(\{1,3\})$, and $C(\{2\})$ can be evaluated yielding the following distance matrices:

$$
\begin{aligned}
& D_{d}^{(1)}=\begin{array}{l}
C(\{1,3\}) \\
C(\{2\})
\end{array}\left[\begin{array}{cc}
C(\{1,3\}) & C(\{2\}) \\
0 & 6 \\
6 & 0
\end{array}\right] \\
& D_{\delta}^{(1)}=\begin{array}{l}
C(\{1,3\}) \\
C(\{2\})
\end{array}\left[\begin{array}{cc}
C(\{1,3\}) & C(\{2\}) \\
0 & 4 \\
4 & 0
\end{array}\right] \\
& D_{\Delta}^{(1)}=\begin{array}{l}
C(\{1,3\}) \\
C(\{2\})
\end{array}\left[\begin{array}{cc}
C(\{1,3\}) & C(\{2\}) \\
0 & 5 \\
5 & 0
\end{array}\right]
\end{aligned}
$$

where the superscript represents the number of updates in the calculation of the distance matrices or equivalently the number of mergings implemented. Note that at the $i$-th update, the distance matrices are of dimension $\left(n_{y}-i\right) \times\left(n_{y}-i\right)$, i.e. they contain $n_{y}-i$ clusters.

The procedure for hierarchical clustering of the input/output pairs described above can be summarized and generalized as follows:

1. At each level of clustering (including the initial optimal decentralized configurations), compute the distance matrices, $D_{d}, D_{\delta}$ and $D_{\Delta}$.

2. Find the smallest off-diagonal value in $D_{d}$ (i.e. the shortest distance between distinct input/output clusters). If there exist multiple pairs of input/output clusters with the smallest value, compare the following values in order:

(a) $\delta$

(b) $\Delta$

Choose the pair with the smallest distance triplet values and merge the clusters to form a new cluster.

3. Repeat steps 1 and 2. Terminate when a single cluster is formed.

Remark 3.1. There is the possibility that there can be multiple pairs of the input/output clusters with the same distance triplet values. In such case, each choice 
can be considered separately, proceeding to the subsequent levels to complete the clustering. Multiple dendrograms will be generated to summarize the results in this case.

Example (continued). Let us consider one of the optimal RDMs obtained for the CSTR example:

$$
M_{r, o p t, 1}=\begin{aligned}
& M \\
& F_{B} \\
& F_{A} \\
& Q
\end{aligned}\left[\begin{array}{cccc}
1 & 2 & 2 & 2 \\
1 & 1 & 1 & 1 \\
1 & 1 & 1 & 1 \\
\infty & 2 & 2 & 1
\end{array}\right]
$$

The initial input/output clusters are formed as follows:

$$
\begin{aligned}
& C(\{1\})=\left\{I O_{1}\right\}=\{F, M\} \\
& C(\{2\})=\left\{I O_{2}\right\}=\left\{F_{B}, c_{A}\right\} \\
& C(\{3\})=\left\{I O_{3}\right\}=\left\{F_{A}, c_{B}\right\} \\
& C(\{4\})=\left\{I O_{4}\right\}=\{Q, T\}
\end{aligned}
$$


The corresponding distance matrices are:

\begin{tabular}{|c|c|c|c|c|c|}
\hline & & $C(\{1\})$ & $C(\{2\})$ & $C(\{3\})$ & $C(\{4\})$ \\
\hline \multirow{4}{*}{$D_{d}=$} & $C(\{1\})$ & 0 & 2 & 2 & $\infty$ \\
\hline & $C(\{2\})$ & 2 & 0 & 0 & 2 \\
\hline & $C(\{3\})$ & 2 & 0 & 0 & 2 \\
\hline & $C(\{4\})$ & $\infty$ & 2 & 2 & 0 \\
\hline \multirow{6}{*}{$D_{\delta}=$} & & $C(\{1\})$ & $C(\{2\})$ & $C(\{3\})$ & $C(\{4\})$ \\
\hline & $C(\{1\})$ & 0 & 0 & 0 & 2 \\
\hline & $C(\{2\})$ & 0 & 0 & 0 & 0 \\
\hline & $C(\{3\})$ & 0 & 0 & 0 & 0 \\
\hline & $C(\{4\})$ & 2 & 0 & 0 & 0 \\
\hline & & $C(\{1\})$ & $C(\{2\})$ & $C(\{3\})$ & $C(\{4\})$ \\
\hline \multirow{4}{*}{$D_{\Delta}=$} & $C(\{1\})$ & 0 & 2 & 2 & $\infty$ \\
\hline & $C(\{2\})$ & 2 & 0 & 1 & 2 \\
\hline & $C(\{3\})$ & 2 & 1 & 0 & 2 \\
\hline & $C(\{4\})$ & $\infty$ & 2 & 2 & 0 \\
\hline
\end{tabular}

The smallest off-diagonal element in $D_{d}$ is $D_{d}(2,3)=D_{d}(3,2)=0$. Thus, we merge $C(\{2\})$ and $C(\{3\})$ to form a new cluster $C(\{2,3\})$. The updated distance 
matrices are:

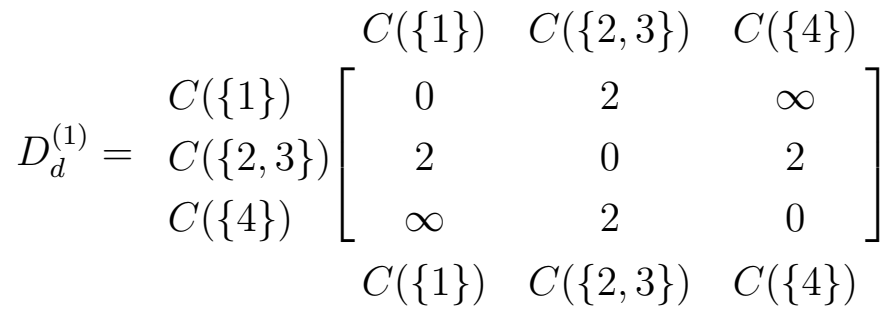

$$
\begin{aligned}
& D_{\delta}^{(1)}=\begin{array}{ll}
C(\{1\}) \\
& C(\{2,3\})
\end{array}\left[\begin{array}{lll}
0 & 0 & 2 \\
0 & 0 & 0 \\
2 & 0 & 0
\end{array}\right] \\
& C(\{1\}) \quad C(\{2,3\}) \quad C(\{4\}) \\
& D_{\Delta}^{(1)}=\begin{array}{l}
C(\{1\}) \\
\\
C(\{2,3\})
\end{array}\left[\begin{array}{ccc}
0 & 2 & \infty \\
2 & 0 & 2 \\
\infty & 2 & 0
\end{array}\right]
\end{aligned}
$$

The distance triplets between $C(\{1\})$ and $C(\{2,3\})$ and between $C(\{2,3\})$ and $C(\{4\})$ are equal to $(2,0,2)$. Therefore, we can choose to merge either, and the results can be summarized as dendrograms shown in Figure 3, along with the distance triplet values between the clusters to be merged at each level of the dendrogram. Note that these triplets are identical for both dendrograms, indicating similar coupling characteristics. We can now draw a horizontal line at any height to cut the dendrogram, resulting in a block decentralized control configuration. The number of intersections between the horizontal line and the vertical lines represent the number of controllers. For example, the dendrogram shown in Figure 3(a) can be cut at a height between $(2,1,2)$ and $(\infty, 2,2)$, to generate a block decentralized control configuration with $\mathrm{IO}_{1}, \mathrm{IO}_{2}, \mathrm{IO}_{3}$ assigned to one controller, and $\mathrm{IO}_{4}$ assigned to the other. Note that such a control configuration is reasonable since the first controller addresses the control of material balance variables, while the energy balance variable is assigned to the other controller.

Remark 3.2. In the case of multiple dendrograms, the distance triplet values at each level are not always the same for all the dendrograms. Different dendrograms can be evaluated noting that larger distance triplet values imply more weakly coupled block decentralized control configurations.

\subsection{Algorithm for automation of synthesis procedure}

The proposed framework can be automated using the algorithm provided below. 
Algorithm: AutomatedControlConfigurationSynthesis

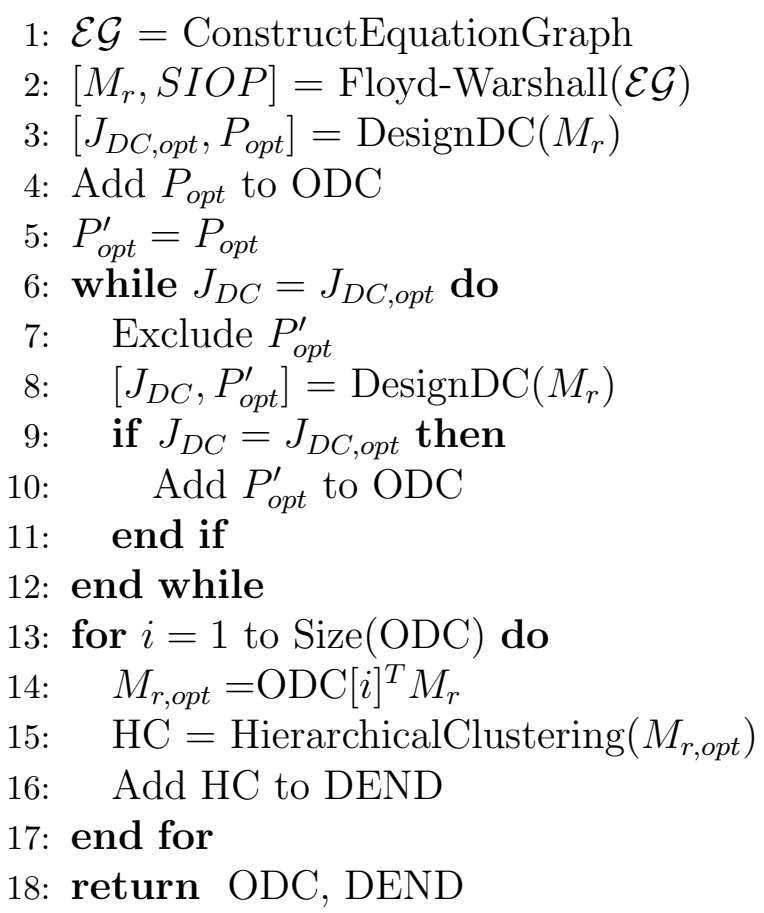

The input to the algorithm is the equations which describe the dynamic behavior of the energy/material balances variables. By default, the material/energy flow rates are marked as the manipulated inputs, and the molar holdups, temperatures and concentrations of the process units are marked as the controlled outputs. An additional input can be provided to the algorithm to define specific sets of manipulated inputs and controlled outputs. The outputs of the algorithm are the optimal decentralized control configurations, and the dendrograms.

The subroutine ConstructEquationGraph configures the equation graph of the given network, $\mathcal{E} \mathcal{G}$, from the input. The RDM of the network $\left(M_{r}\right)$, and the shortest IOPs between all input/output pairs (SIOP) are computed using the Floyd-Warshall algorithm (Leiserson et al., 2001). The Floyd-Warshall algorithm initially sets the length of the path between different nodes to 1 if the nodes are directly connected, otherwise to a very large finite integer, which, in our case, is chosen to be 10001, so that the relative degree of $10^{4}$ represents a relative degree of infinity.

The subroutine DesignDC computes the optimal objective function value, $J_{D C \text {,opt }}$, and $P_{\text {opt }}$ by solving the optimization problem defined by Eqs.(7)-(11). $P_{\text {opt }}$ is added to 
$O D C$, the set of optimal decentralized control configurations. The while loop is added to find the alternative solutions. Then, for each configuration in $O D C$, hierarchy of input/output clusters $H C$, which is built in a form of dendrogram, is generated using the subroutine HierarchicalClustering which automates the procedure proposed in the previous section. $H C$ is added to $D E N D$, the set of dendrograms.

In what follows, we illustrate the application of the above algorithm using an example process network.

\section{Case study - energy integrated solid oxide fuel cell (SOFC) system}

\subsection{Process description}

Let us consider the energy integrated SOFC system with an external reformer proposed in Georgis et al. (2011). The process flow diagram of the system is shown in Figure 4. In this system, the feed stream of water is vaporized in HE1 and HE2, and it is mixed with the methane stream which is preheated in HE7. Then, the fuel mixture is heated in HE3 before entering the steam reformer to facilitate the following reactions:

$$
\begin{aligned}
\mathrm{CH}_{4}+\mathrm{H}_{2} \mathrm{O} & \rightleftharpoons \mathrm{CO}+3 \mathrm{H}_{2} \\
\mathrm{CO}+\mathrm{H}_{2} \mathrm{O} & \rightleftharpoons \mathrm{CO}_{2}+\mathrm{H}_{2}
\end{aligned}
$$

The outlet of the steam reformer is heated in HE5, and supplied into the anode of the fuel cell. The air feed stream is heated in HE4 and HE6, then fed into the cathode of the fuel cell. In the fuel cell, electrochemical potential is generated through the following redox reactions:

$$
\begin{aligned}
\mathrm{H}_{2}+\mathrm{O}^{2-} & \rightarrow \mathrm{H}_{2} \mathrm{O}+2 \mathrm{e}^{-} \\
\frac{1}{2} \mathrm{O}_{2}+2 \mathrm{e}^{-} & \rightarrow \mathrm{O}^{2-}
\end{aligned}
$$

Both the outlet streams of the fuel cell are then fed into the catalytic burner where methane, carbon monoxide and hydrogen are completely combusted. The outlet stream of the catalytic burner, which is at high temperature, provides energy to the stream reformer and the feed streams as it passes through the jacket of the stream reformer and the heat exchangers.

Important control objectives for this system are:

- control the temperature of the fuel cell $\left(y_{1}\right)$ to prevent material damages while sustaining the ioninc conductivity of the electrolyte 
- control the fuel utilization of the fuel cell $\left(y_{2}\right)$ to ensure efficient usage of fuel while preventing fuel starvation

- control the fuel cell air inlet temperature $\left(y_{3}\right)$ to enhance the operation of the fuel cell

- control the conversion of the steam reformer $\left(y_{4}\right)$ to generate hydrogen-rich stream

- control the inlet temperature of the steam reformer $\left(y_{5}\right)$ to facilitate the reactions

Also, we have the following potential manipulated inputs:

- the flow rate of air stream entering the fuel cell $\left(u_{1}\right)$

- the flow rate of fuel stream entering the fuel cell $\left(u_{2}\right)$

- the duty of HE6

- the jacket flow rate of the steam reformer $\left(u_{4}\right)$

- the duty of HE3

Since the duty of heat exchangers cannot be manipulated directly, the bypass ratio around the hot channel of HE6 $\left(u_{3}\right)$ and the bypass ratio around the hot channel of HE3 $\left(u_{5}\right)$ will be used as manipulated inputs.

\subsection{Automated synthesis of control configurations}

Let us now apply the developed graph-theoretic framework to this energy integrated SOFC system. The original equation graph consists of 224 nodes and 529 edges, and for brevity, only a part of the original equation graph, which contains the nodes for the inputs and the outputs mentioned previously, and the nodes for the state variables that are included in the IOPs, is shown in Figure 5. Table 2 shows the list of the nodes in this graph. The RDM is obtained as:

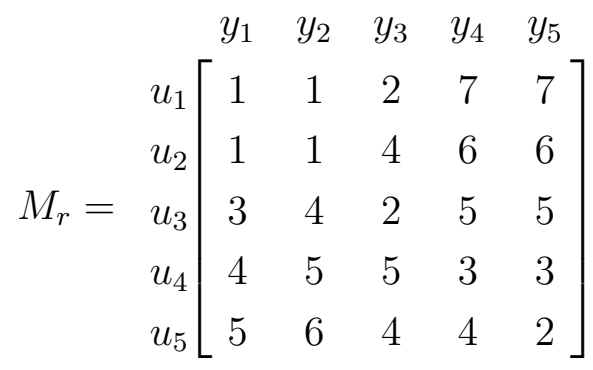


Two optimal solutions are returned by the algorithm:

$$
\begin{aligned}
P_{o p t, 1}= & {\left[\begin{array}{lllll}
1 & 0 & 0 & 0 & 0 \\
0 & 1 & 0 & 0 & 0 \\
0 & 0 & 1 & 0 & 0 \\
0 & 0 & 0 & 1 & 0 \\
0 & 0 & 0 & 0 & 1
\end{array}\right] } \\
P_{o p t, 2}= & {\left[\begin{array}{lllll}
0 & 1 & 0 & 0 & 0 \\
1 & 0 & 0 & 0 & 0 \\
0 & 0 & 1 & 0 & 0 \\
0 & 0 & 0 & 1 & 0 \\
0 & 0 & 0 & 0 & 1
\end{array}\right] }
\end{aligned}
$$

which translate into the following optimal RDMs:

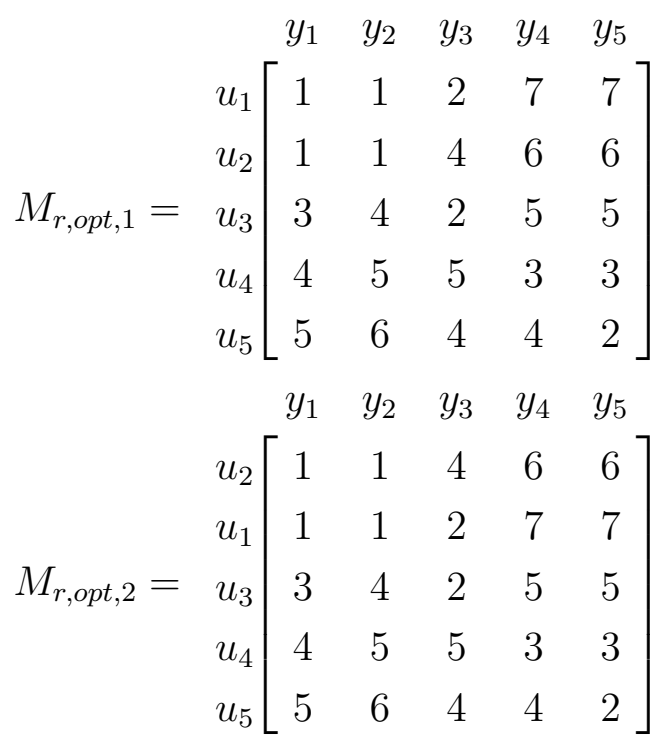

From both solutions, we note that, for each row, the diagonal element has the smallest value, implying that each input is paired with the output that it has the most direct effect on. The process flow diagram with the control loops determined by $P_{\text {opt }, 1}$ is shown in Figure 6. In this configuration, the temperature and the fuel utilization of the fuel cell are controlled by manipulating the flow rates of the air and fuel inlet stream, respectively, and the air inlet temperature is controlled by manipulating the bypass ratio around the hot channel of HE6. The jacket flow rate of the steam reformer and the bypass ratio around the hot channel of HE3 are manipulated to control the conversion and the inlet temperature of the steam reformer. Note that 
this control configuration matches the control strategy proposed and evaluated via simulations in Georgis et al. (2011). By switching the manipulated inputs which are used to control the temperature and the fuel ulitization of the fuel cell in the previous configuration, we get the control configuration determined by $P_{o p t, 2}$. This change is reasonable since $u_{1}$ and $u_{2}$ have the same structural effect on $y_{1}$ and $y_{2}$ (i.e. $\left.M_{r}(1,1)=M_{r}(1,2)=M_{r}(2,1)=M_{r}(2,2)=1\right)$.

For the control configuration determined by $P_{o p t, 1}$, we have the following initial clusters:

$$
\begin{aligned}
& C(\{1\})=I O_{1}=\left\{u_{1}, y_{1}\right\} \\
& C(\{2\})=I O_{2}=\left\{u_{2}, y_{2}\right\} \\
& C(\{3\})=I O_{3}=\left\{u_{3}, y_{3}\right\} \\
& C(\{4\})=I O_{4}=\left\{u_{4}, y_{4}\right\} \\
& C(\{5\})=I O_{5}=\left\{u_{5}, y_{5}\right\}
\end{aligned}
$$

and the distance matrix $D_{d}$ :

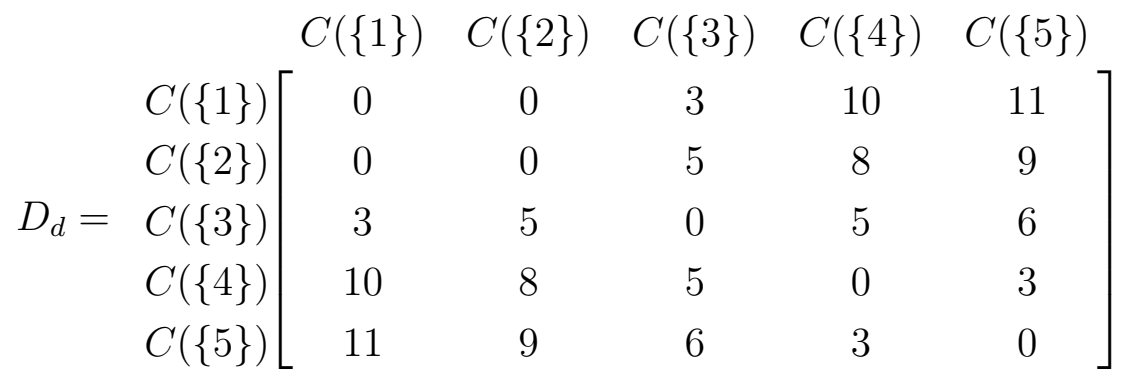

Note that there is only one pair of clusters with the shortest distance $(C(1)$ and $C(2)$ ), thus the additional criteria are not required at this level. $C(\{1\})$ and $C(\{2\})$ are the clusters to be merged, and $D_{d}$ is updated accordingly:

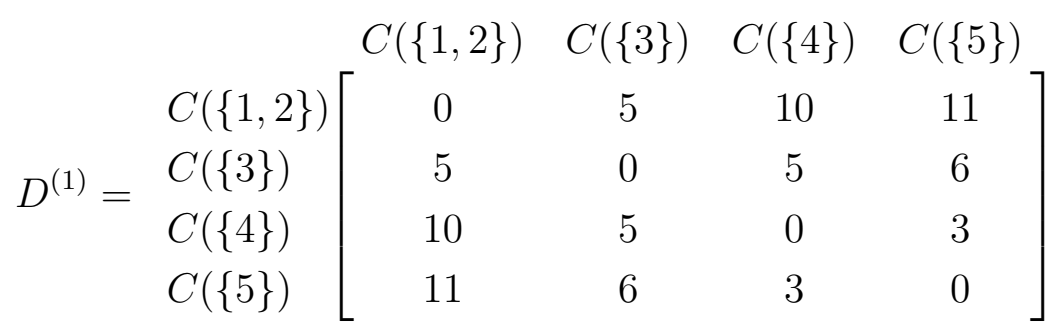

Again, we have only one pair of clusters with the shortest distance, $C(\{4\})$ and 
$C(\{5\})$, which are merged yielding the following distance matrix:

$$
\begin{array}{rlcc} 
& C(\{1,2\}) & C(\{3\}) & C(\{4,5\}) \\
D^{(2)}= & C(\{1,2\}) & C(\{3\}) \\
& C(\{4,5\})
\end{array}\left[\begin{array}{ccc}
0 & 5 & 11 \\
5 & 0 & 6 \\
11 & 6 & 0
\end{array}\right]
$$

We finally merge $C(\{1,2\})$ and $C(\{3\})$, and then $C(\{1,2,3\})$ and $C(\{4,5\})$ to complete the clustering. Note that, in this case, the additional criteria are not used as we have only one pair of clusters with the shortest distance at each level of clustering. The hierarchical clustering results obtained from $M_{r, o p t, 1}$ and $M_{r, o p t, 2}$ are shown in Figures 7 and 8, respectively.

The dendrograms shown in both figures can be cut at different heights to generate the block decentralized control configurations summarized in Tables 3 and 4, respectively. We can compare the block decentralized configurations at the same level (e.g. solution 1-1 and solution 2-1) based on the distance triplet values shown in Figures 7 and 8. In solution 1-1 and solution 2-1, the distance triplets of the closest clusters are $(3,1,4)$. Thus, the block decentralized control configurations in both solutions are equally good from a structural point of view. In solution 1-2 and solution 2-2, the distance triplets of the closest clusters are $(5,5,4)$ and $(5,3,4)$, respectively. Since solution 1-2 has a greater $\delta$, it represents a more weakly coupled control configuration than solution 2-2. Similarly, the control configuration in solution 2-3 is more weakly coupled than the one in solution 1-3. Note that, although the additional criteria (i.e. $\delta$ and $\Delta$ ) are not used to complete the clustering, they can be used to further analyze the results.

Noting that the manipulated inputs and the controlled outputs represent physical properties of process units and the flows connecting them, the hierarchical clustering results can also be used to identify subnetworks of closely coupled and decoupled process units. By identifying the process units associated with the inputs and outputs in each cluster, different subnetworks are detected as shown in Figure 9. For example, as shown in Figure 10, solution 1-3 suggests to address the control problem around the fuel cell (i.e. control of the temperature, the fuel utilization and the air inlet temperature of the fuel cell) using a MIMO controller with the flow rates of the air and fuel inlet streams, and the bypass ratio around the hot channel of HE6 as the manipulated inputs, while the control problem around the steam reformer (i.e. control of the temperature and the conversion of the steam reformer) is addressed by another MIMO controller manipulating the jacket inlet flowrate and the bypass ratio around the hot channel of HE3. These choices look reasonable but of course will need to be evaluated using additional criteria as well, and ultimately via simulations. 


\section{Conclusions}

In this paper, a method for optimal control configuration synthesis based on structural coupling (physical closeness) has been developed. Specifically, we proposed an integer optimization problem formulation to synthesize optimal decentralized control configurations using the relative degree as a measure of structural coupling. Then, we proposed a hierarchical clustering procedure to identify groups of inputs and outputs such that the variables in the same group interact strongly with each other, while different groups are loosely coupled. This was based on a distance metric defined between input/output clusters, and additional distance measures to differentiate among clusters with the same distance. The proposed method was automated within a graph theoretic framework and was illustrated through an example process network. This procedure can be used to generate block decentralized control configuration candidates, to be further evaluated by the control designer. The proposed framework can be further developed to incorporate i) additional process information such as gains and time constants, and ii) heuristics so that specific pairings are included in or excluded from the optimal control configuration.

\section{Acknowledgements}

Financial support for this work by the National Science Foundation, grant CBET1133167, is gratefully acknowledged. The authors thank Dimitrios Georgis for his helpful insights on the case study, and Sujit Jogwar and Srinivas Rangarajan for the helpful discussions during the course of this research.

\section{References}

Baldea, M., Araujo, A., Skogestad, S., Daoutidis, P., 2008. Dynamic considerations in the synthesis of self-optimizing control structures. AIChE J. 54 (7), 1830-1841.

Baldea, M., Daoutidis, P., Kumar, A., 2006. Dynamics and control of integrated networks with purge streams. AIChE J. 52 (4), 1460-1472.

Chang, J., Yu, C., 1990. The relative gain for non-square multivariable systems. Chem. Eng. Sci. 45 (5), 1309-1323.

Christofides, P., Davis, J., El-Farra, N., Clark, D., Harris, K., Gipson, J., 2007. Smart plant operations: Vision, progress and challenges. AIChE J. 53 (11), 2734-2741.

Daoutidis, P., Kravaris, C., 1992. Structural evaluation of control configurations for multivariable nonlinear processes. Chem. Eng. Sci. 47 (5), 1091-1107. 
Ellis, M., Christofides, P., 2014. Selection of control configurations for economic model predictive control systems. AIChE J. 60 (9), 3230-3242.

Georgis, D., Jogwar, S., Almansoori, A., Daoutidis, P., 2011. Design and control of energy integrated SOFC systems for in situ hydrogen production and power generation. Comput. Chem. Eng. 35 (9), 1691-1704.

Girvan, M., Newman, M., 2002. Community structure in social and biological networks. P. Natl. Acad. Sci. 99 (12), 7821-7826.

Heath, J., Kookos, I., Perkins, J., 2000. Process control structure selection based on economics. AIChE J. 46 (10), 1998-2016.

Hudon, N., Bao, J., 2012. Dissipativity-based decentralized control of interconnected nonlinear chemical processes. Comput. Chem. Eng. 45, 84-101.

IBM, 2011. IBM ILOG CPLEX Optimization Studio 12.2.

Jiang, Z., Zhou, W., Xu, B., Yuan, W., 2007. Process flow diagram of an ammonia plant as a complex network. AIChE J. 53 (2), 423-428.

Jogwar, S., Baldea, M., Daoutidis, P., 2009. Dynamics and control of process networks with large energy recycle. Ind. Eng. Chem. Res. 48 (13), 6087-6097.

Kariwala, V., Forbes, J., Meadows, E., 2003. Block relative gain: Properties and pairing rules. Ind. Eng. Chem. Res. 42 (20), 4564-4574.

Khalil, H., 2002. Nonlinear systems. Vol. 3. Prentice hall Upper Saddle River.

Kumar, A., Daoutidis, P., 2002. Nonlinear dynamics and control of process systems with recycle. J. Process Contr. 12 (4), 475-484.

Kunen, K., Vaughan, J., 1984. Handbook of set-theoretic topology. North Holland, Amsterdam.

Leiserson, C., Rivest, R., Stein, C., Cormen, T., 2001. Introduction to algorithms. The MIT press.

Liu, J., Muñoz de la Peña, D., Christofides, P., 2009. Distributed model predictive control of nonlinear process systems. AIChE J. 55 (5), 1171-1184.

Luyben, M., Tyreus, B., Luyben, W., 1997. Plantwide control design procedure. AIChE J. 43 (12), 3161-3174. 
Manousiouthakis, V., Savage, R., Arkun, Y., 1986. Synthesis of decentralized process control structures using the concept of block relative gain. AIChE J. 32 (6), 9911003.

Mhaskar, P., Gani, A., McFall, C., Christofides, P., Davis, J., 2007. Fault-tolerant control of nonlinear process systems subject to sensor faults. AIChE J. 53 (3), 654-668.

Newman, M., 2004. Detecting community structure in networks. Eur. Phys. J. B 38 (2), 321-330.

Newman, M., 2006. Modularity and community structure in networks. P. Natl. Acad. Sci. 103 (23), 8577-8582.

Ng, C., Stephanopoulos, G., 1996. Synthesis of control systems for chemical plants. Comput. Chem. Eng. 20, S999-S1004.

Price, R., Georgakis, C., 1993. Plantwide regulatory control design procedure using a tiered framework. Ind. Eng. Chem. Res. 32 (11), 2693-2705.

Psaltis, A., Kookos, I., Kravaris, C., 2013. Plant-wide control structure selection methodology based on economics. Comput. Chem. Eng. 52, 240-248.

Psaltis, A., Kookos, I., Kravaris, C., 2014. Plantwide control structure selection methodology for the benchmark vinyl acetate monomer plant. Comput. Chem. Eng. 62, 108-116.

Rangaiah, G., Kariwala, V., 2012. Plantwide control: Recent developments and applications. John Wiley \& Sons.

Rawlings, J., Stewart, B., 2008. Coordinating multiple optimization-based controllers: New opportunities and challenges. J. Process Contr. 18 (9), 839-845.

Scattolini, R., 2009. Architectures for distributed and hierarchical model predictive control-a review. J. Process Contr. 19 (5), 723-731.

Schné, T., Hangos, K., 2011. Decentralised controller structure design and retrofit of process systems based on graph theory. Int. J. Syst. Sci. 42 (6), 1023-1033.

Skogestad, S., 2000a. Plantwide control: The search for the self-optimizing control structure. J. Process Contr. 10 (5), 487-507. 
Skogestad, S., 2000b. Self-optimizing control: The missing link between steady-state optimization and control. Comput. Chem. Eng. 24 (2), 569-575.

Sun, Y., El-Farra, N., 2008. Quasi-decentralized model-based networked control of process systems. Comput. Chem. Eng. 32 (9), 2016-2029.

Tippett, M., Bao, J., 2013. Distributed model predictive control based on dissipativity. AIChE J. 59 (3), 787-804.

van de Wal, M., de Jager, B., 2001. A review of methods for input/output selection. Automatica 37 (4), 487-510.

Varigonda, S., Kalmár-Nágy, T., LaBarre, B., Mezic, I., 2004. Graph decomposition methods for uncertainty propagation in complex, nonlinear interconnected dynamical systems. In: Decision and Control (CDC), 2004 43rd IEEE Conference on. Vol. 2. IEEE, pp. 1794-1798.

Ydstie, B., 2002. Passivity based control via the second law. Comput. Chem. Eng. 26 (7), 1037-1048. 


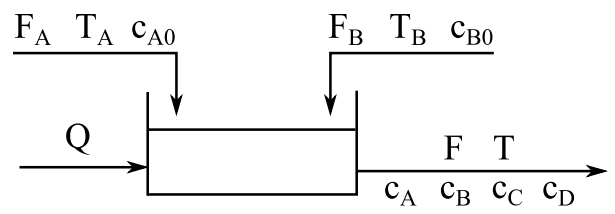

Figure 1: A simple CSTR 


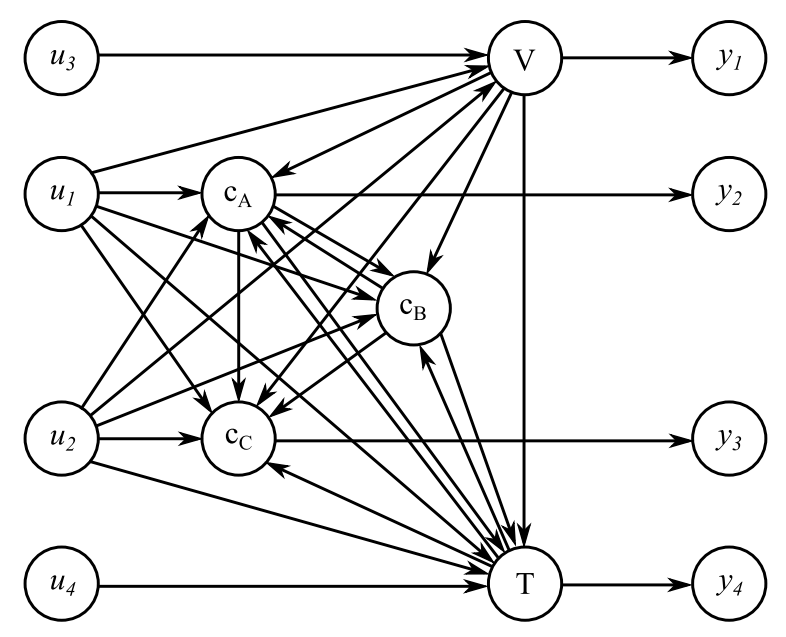

Figure 2: Equation graph of the simple CSTR example 


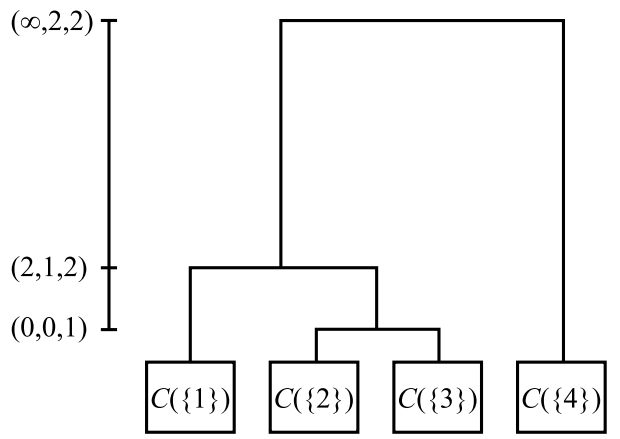

(a)

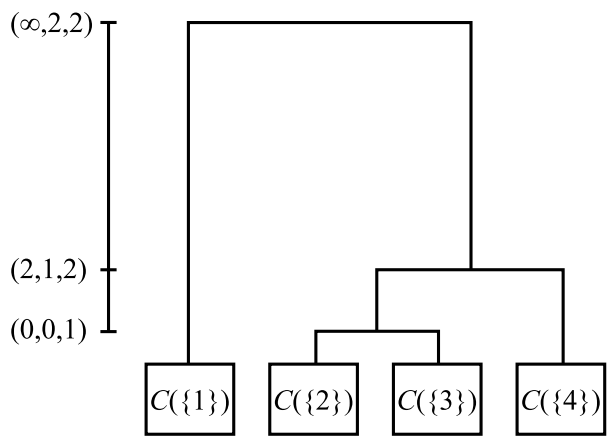

(b)

Figure 3: Hierarchical clustering results for the simple CSTR example 


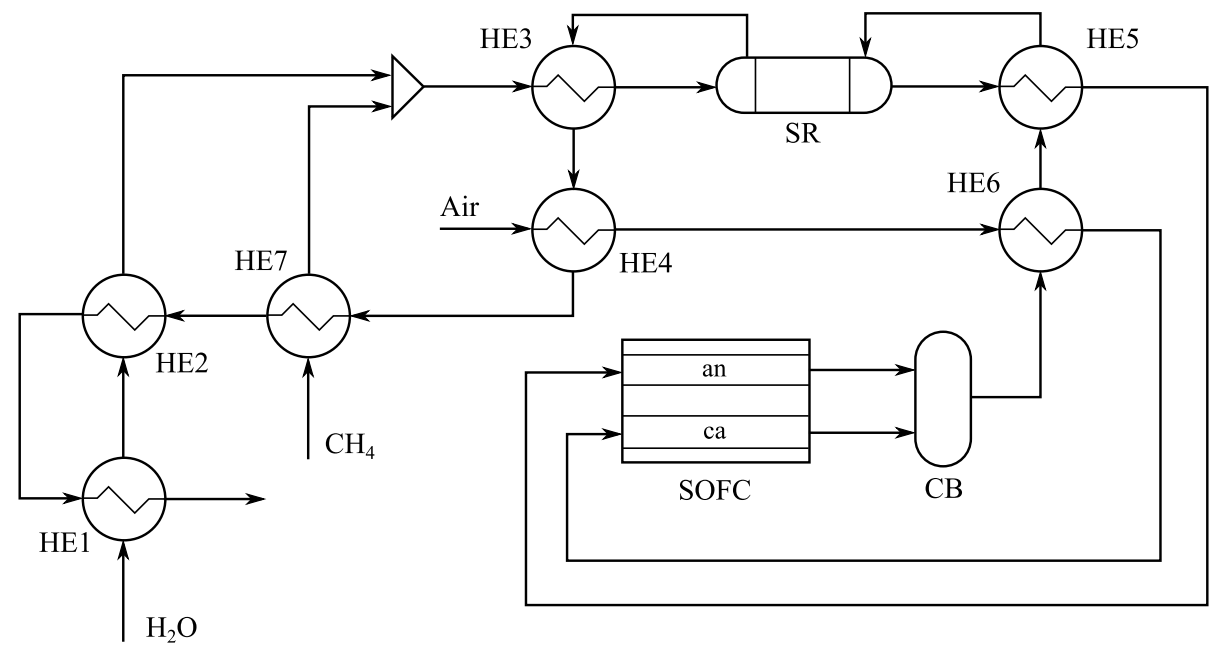

Figure 4: Process flow diagram of the energy integrated SOFC system 


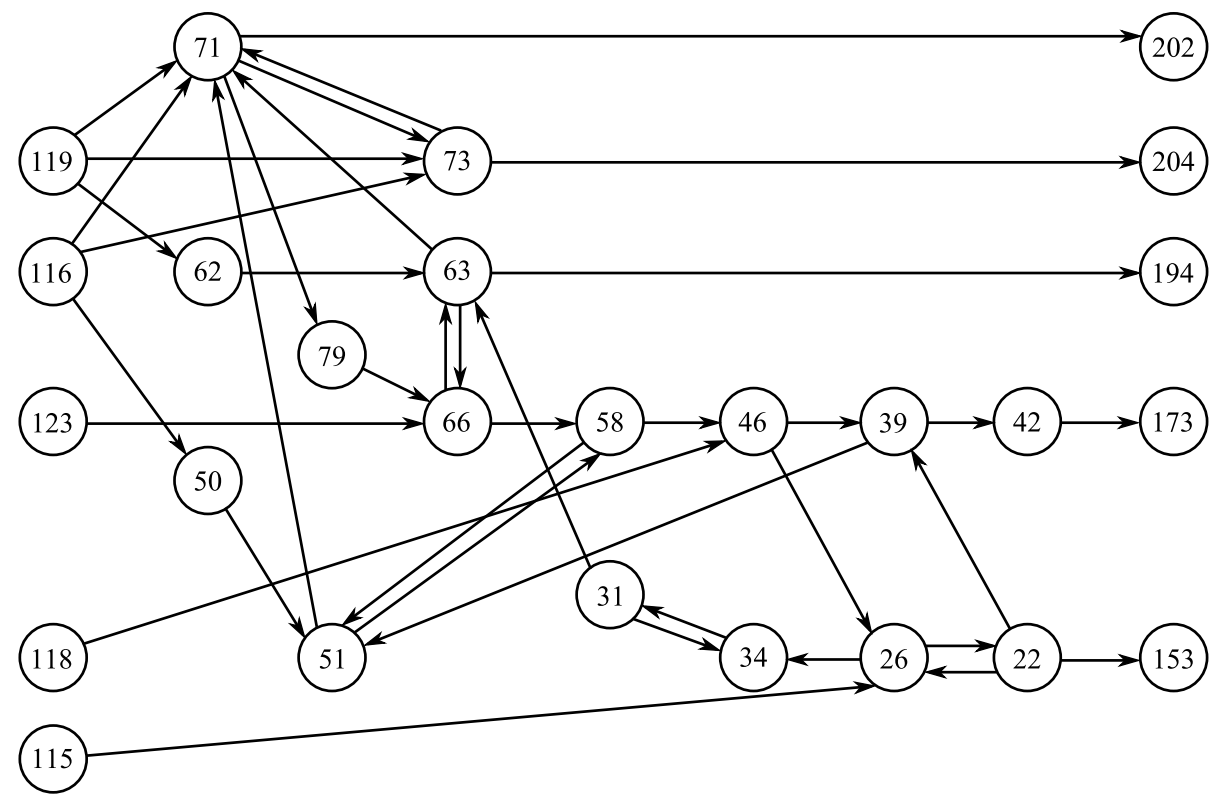

Figure 5: Part of the equation graph of the energy integrated SOFC system 


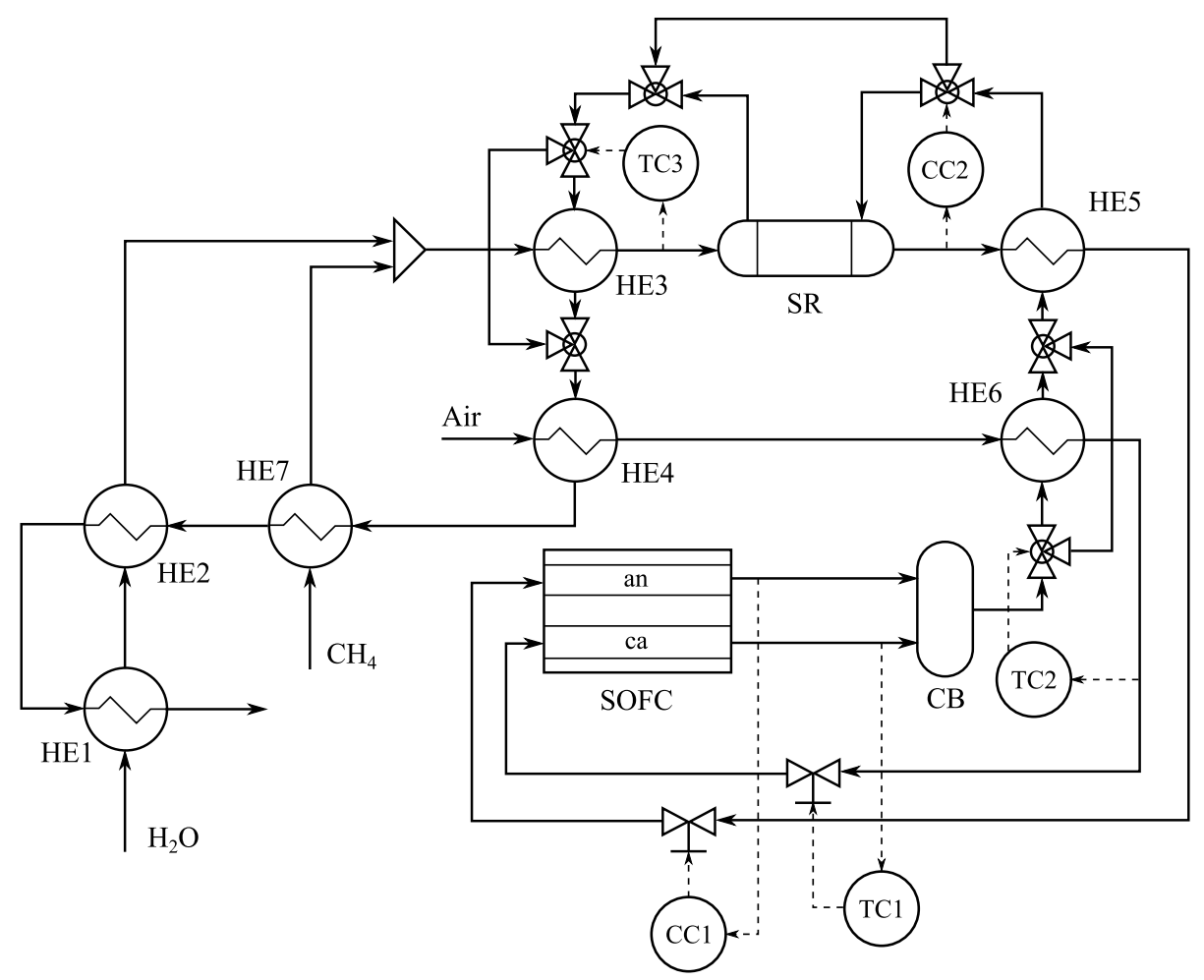

Figure 6: Control configuration for solution 1 


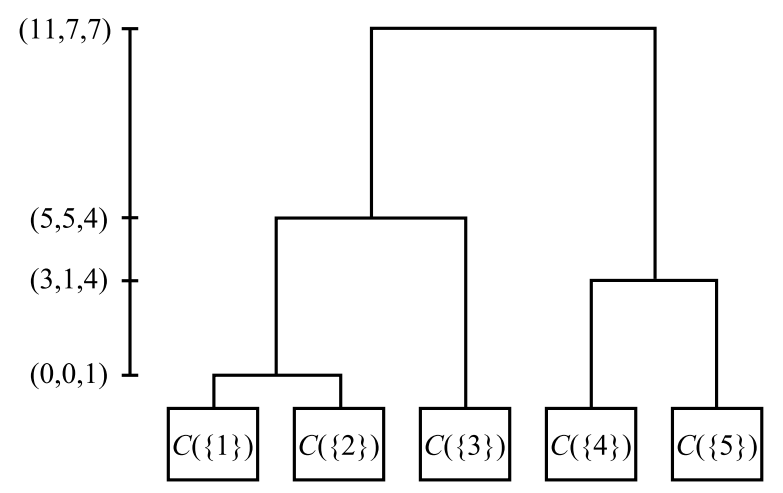

Figure 7: Hierarchical clustering result for the energy integrated SOFC system, solution 1 


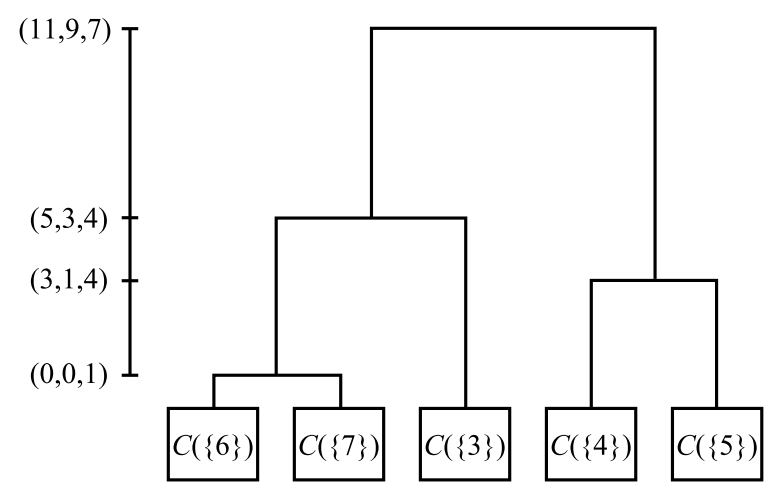

Figure 8: Hierarchical clustering result for the energy integrated SOFC system, solution $2(C(\{6\})=$ $\left.\left\{u_{2}, y_{1}\right\}, C(\{7\})=\left\{u_{1}, y_{2}\right\}\right)$ 


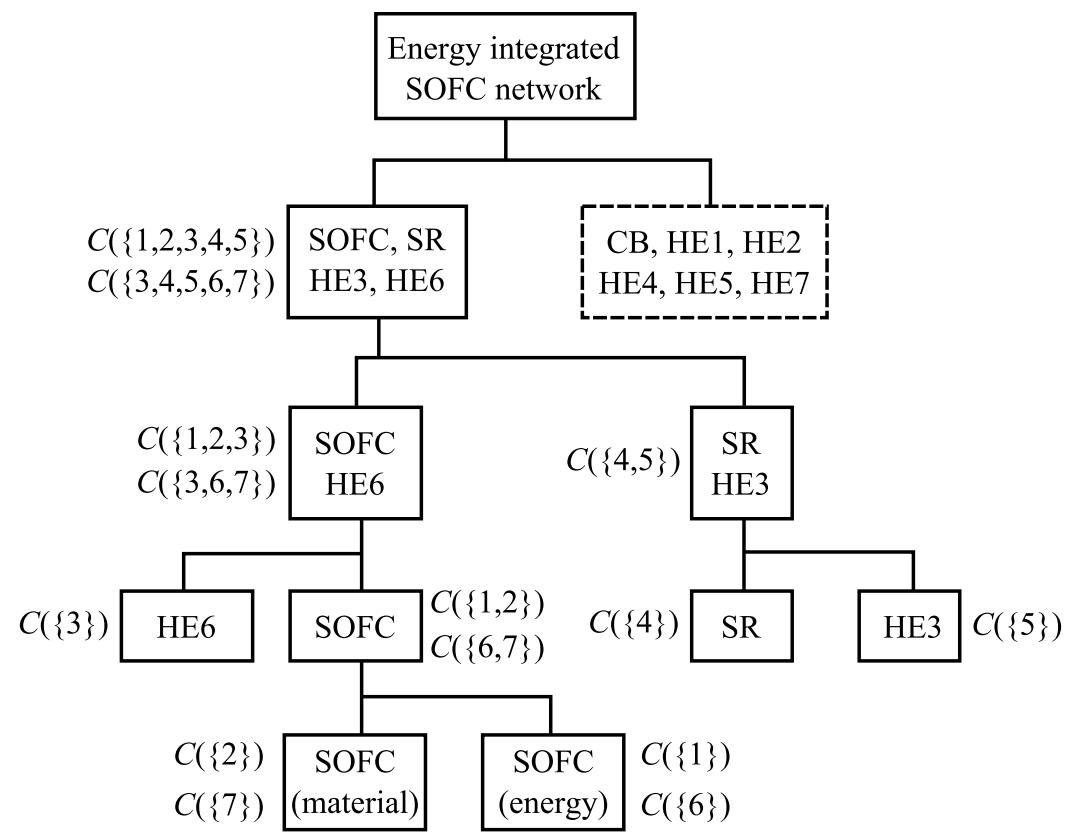

Figure 9: Subnetworks of the energy integrated SOFC system 


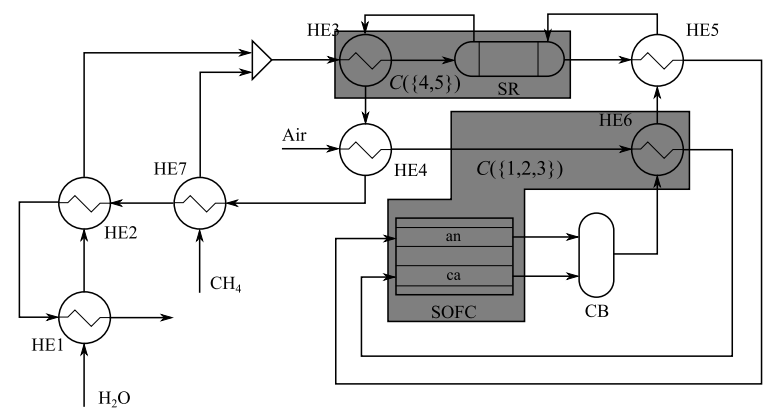

Figure 10: Subnetworks controlled by different controllers, solution 1-3 


\begin{tabular}{|c|cccc|}
\hline & $y_{1}(M)$ & $y_{2}\left(c_{A}\right)$ & $y_{3}\left(c_{C}\right)$ & $y_{4}(T)$ \\
\hline$u_{1}\left(F_{A}\right)$ & $F_{A} \rightarrow M \rightarrow y_{1}$ & $F_{A} \rightarrow c_{A} \rightarrow y_{2}$ & $F_{A} \rightarrow c_{C} \rightarrow y_{3}$ & $F_{A} \rightarrow T \rightarrow y_{4}$ \\
$u_{2}\left(F_{B}\right)$ & $F_{B} \rightarrow M \rightarrow y_{1}$ & $F_{B} \rightarrow c_{A} \rightarrow y_{2}$ & $F_{B} \rightarrow c_{C} \rightarrow y_{3}$ & $F_{B} \rightarrow T \rightarrow y_{4}$ \\
$u_{3}(F)$ & $F \rightarrow M \rightarrow y_{1}$ & $F \rightarrow M \rightarrow c_{A} \rightarrow y_{2}$ & $F \rightarrow M \rightarrow c_{C} \rightarrow y_{3}$ & $F \rightarrow M \rightarrow T \rightarrow y_{4}$ \\
$u_{4}(Q)$ & No path & $Q \rightarrow T \rightarrow c_{A} \rightarrow y_{2}$ & $Q \rightarrow T \rightarrow c_{C} \rightarrow y_{2}$ & $Q \rightarrow T \rightarrow y_{4}$ \\
\hline
\end{tabular}

Table 1: Shortest paths between the inputs and the outputs of the simple CSTR example 


\begin{tabular}{|cc|cc|}
\hline \multicolumn{4}{|c|}{ Input nodes } \\
\hline Index & Variable & Index & Variable \\
\hline 115 & $u_{5}$ & 119 & $u_{1}$ \\
116 & $u_{2}$ & 123 & $u_{3}$ \\
118 & $u_{4}$ & \\
\hline \multicolumn{4}{|c|}{ State nodes } \\
\hline Index & Variable & Index & Variable \\
\hline 22 & $T_{H E 3, \text { cold }}$ & 51 & $T_{H E 5, \text { cold }}$ \\
26 & $T_{H E 3, \text { hot }}$ & 58 & $T_{H E 5, \text { hot }}$ \\
31 & $T_{H E 4, \text { cold }}$ & 62 & $M_{H E 6, \text { cold }}$ \\
34 & $T_{H E 4, \text { hot }}$ & 63 & $T_{H E 6, \text { cold }}$ \\
39 & $T_{S R}$ & 66 & $T_{H E 6, \text { hot }}$ \\
42 & $c_{C H}, S R$ & 71 & $T_{S O F C}$ \\
46 & $T_{S R J}$ & 73 & $c_{H_{2}, S O F C}$ \\
50 & $M_{H E 5, \text { cold }}$ & 79 & $T_{C B}$ \\
\hline \multicolumn{4}{|c|}{ Output nodes } \\
\hline Index & Variable & Index & Variable \\
\hline 153 & $y_{5}$ & 202 & $y_{1}$ \\
173 & $y_{4}$ & 204 & $y_{2}$ \\
194 & $y_{3}$ & \\
\hline
\end{tabular}

Table 2: Node list of the energy integrated SOFC system equation graph in Figure 5 


\begin{tabular}{|ccc|}
\hline Solution & Number of Controller & Input/output clusters \\
\hline $1-1$ & 4 & $C(\{1,2\}), C(\{3\}), C(\{4\}), C(\{5\})$ \\
$1-2$ & 3 & $C(\{1,2\}), C(\{3\}), C(\{4,5\})$ \\
$1-3$ & 2 & $C(\{1,2,3\}), C(\{4,5\})$ \\
\hline
\end{tabular}

Table 3: Block decentralized control configurations generated from the energy integrated SOFC system, solution 1 


\begin{tabular}{|ccc|}
\hline Solution & Number of Controller & Input/output clusters \\
\hline $2-1$ & 4 & $C(\{6,7\}), C(\{3\}), C(\{4\}), C(\{5\})$ \\
$2-2$ & 3 & $C(\{6,7\}), C(\{3\}), C(\{4,5\})$ \\
$2-3$ & 2 & $C(\{3,6,7\}), C(\{4,5\})$ \\
\hline
\end{tabular}

Table 4: Block decentralized control configurations generated from the energy integrated SOFC system, solution 2 